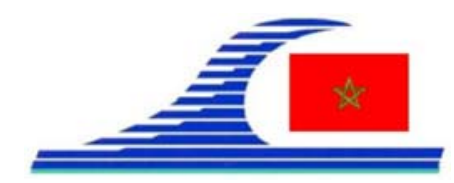

Conférence Méditerranéenne Côtière et Maritime

EDITION 2, TANGER, MAROC (2011)

Coastal and Maritime Mediterranean Conference

Disponible en ligne - http://www.paralia.fr - Available online

\title{
Contrôle et évolution des milieux écologiques de la lagune de Nador (Maroc)
}

\author{
Naima HAMOUMI ${ }^{1}$, Touria HSSAIDA ${ }^{2}$, Loubna TERHZAZ ${ }^{1}$, \\ Mohammed El Amine HAZIM ${ }^{1}$
}

1. Laboratoire Océanologie et Géodynamique des Bassins sédimentaires, Faculté des Sciences, Université Mohammed V - Agdal, Rabat, Maroc.naimahamoumi@yahoo.fr 2. Dépt. de Géologie, Fac. des Sciences, U. Hassan II Ben Mssik, Casablanca, Maroc.

\section{Résumé :}

L'étude écologique lagune de Nador (littoral méditerranéen oriental) a porté sur l'étude quantitative et qualitative de deux groupes de microfossiles qui ont un intérêt écologique particulier : les foraminifères benthiques et les ostracodes. Pour ce faire, une démarche nouvelle a été adoptée elle est basée sur : (1) l'application pour la première fois de la méthode des courbes des fréquences relatives aux foraminifères et aux ostracodes pour l'identification des milieux écologiques et (2) l'étude de l'évolution spatiale des milieux écologiques pour la période actuelle (hiver 2003 et été 2004) et pour une période estimée à 1000-1200 ans BP.

Elle a permis de reconstituer les milieux écologiques, de suivre leur évolution spatiale pour l'actuel (hiver 2003, été 2004) et pour une période ancienne (1000 à 1200 ans BP) et de préciser les facteurs naturels (climat, géomorphologie, morphodynamique et hydrologie) et les facteurs anthropiques qui contrôlent leur répartition et leur évolution. Par ailleurs, cette étude a mis en évidence l'existence d'une relation étroite entre l'évolution des ostracodes et celle de la famille des Spiroloculinidae qui pourraient constituer des bio indicateurs de la pollution.

Mots-clés : Ecosystème côtier - Ecologie - Foraminifères benthiques - Ostracodes Bio indicateurs de la pollution - Lagune de Nador- Maroc - Littoral méditerranéen

\section{Introduction}

La lagune de Nador qui est parmi les zones les plus productives de la façade méditerranéenne, joue un rôle économique important dans la région (pêche, aquaculture). De ce fait et du fait de sa situation en bordure des centres urbains de Nador, Beni Ensar et Kariat Arekmane, elle a été soumise à une pression anthropique importante et souvent anarchique. Il était donc intéressant d'entreprendre dans le cadre du projet européen COLASU une étude des foraminifères benthique et des ostracodes (bio indicateurs des écosystèmes lagunaires) afin de reconstituer les milieux écologiques et les facteurs qui les contrôlent. Et ce d'autant plus que les travaux précédents (IRZI, 1987 et 2002), d’une part ne précisent pas les fréquences des ostracodes et d'autre part, ne distinguent pas les associations de foraminifères des bases et des sommets des 
La connaissance de la Mer :

un vecteur du développement durable en Méditerranée

carottes, qui appartiennent pourtant à des périodes différentes séparées d'au moins 1000 ans, pour la reconstitution des zones écologiques. Par ailleurs, les autres études réalisées dans le cadre du projet COLASU, ont porté dans le cas des foraminifères benthiques (GONZALEZ-REGALADO et al., 2005) sur 4 stations des sommets de carottes de l'hiver 2003, différentes de celles étudiées dans ce travail et dans le cas des ostracodes (RUIZ et al., 2004) sur 12 stations des sommets de carottes de l'hiver 2003 dont trois seulement coïncident avec celles étudiées dans ce travail.

\section{Matériels et méthodes}

L'étude quantitative et qualitative des foraminifères benthiques et des ostracodes (BOUIMETARHAN, 2004) a porté sur 17 échantillons pris dans les sommets et les bases de 9 carottes prélevées au cours de l'hiver 2003 et sur 9 échantillons pris dans les sommets des carottes prélevées durant l'été 2004 (Fig. 1). Cette étude a été menée en parallèle avec celles de l'étude des paramètres environnementaux, de la bathymétrie, des sédiments et de la géomorphologie et la morphoynamique de lagune de Nador. Par ailleurs, une démarche nouvelle a été adoptée (HAMOUMI et al., 2005), elle est basée sur : 1) l'application pour la première fois de la méthode des courbes des fréquences relatives aux foraminifères et aux ostracodes pour l'identification des milieux écologiques et 2) l'étude de l'évolution spatiale des milieux écologiques pour la période actuelle (hiver 2003 et été 2004) et pour une période estimée à 1000 - 1200 ans BP d'après les datations de MAHJOUBI (2001).

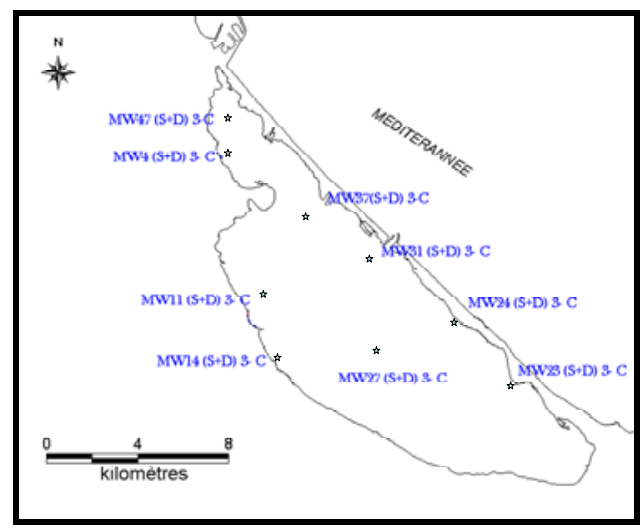

Figure 1: Situation des stations de carottage (campagnes : hiver 2003 et été 2004).

\section{Résultats}

Trois milieux écologiques (I, II et III) ont été identifiés pour l’Hiver 2003 (Fig. 2 A). Le milieu écologique I correspond à la bordure continentale nord. Il se caractérise par : 1) une association d'ostracodes qui montre des proportions voisines de Cyprideis (40.1\%) et de Loxoconcha (37.7\%), une faible densité et une biodiversité assez élevée et 2) une association de foraminifères où prédominent les formes péritidales, en particulier Ammonia tepida (68\%). Le milieu écologique II (confiné) correspond à l'extrémité NW 
et la bordure continentale sud. Il se caractérise par : 1) une association d'ostracodes où Loxoconcha (> 50\%) prédomine sur Cyprideis (20 à 30\%), une faible densité et une biodiversité en général moyenne, et 2) une association de foraminifères dominée par le groupe peritidal où la famille des Nonionidae (Ammonia tepida suivie de Nonion depressulum), atteint un pourcentage de $60 \%$ et comportant des espèces marines épifauniques (Miliolidae et Bolivinidae). Le milieu écologique III (sous influence marine) correspond à la bordure interne de l'île barrière, la partie centrale et la zone de la passe. Il se caractérise par : 1) une association d'ostracodes où Cyprideis (>60\%) prédomine sur Loxoconcha (10 à 20\%) et généralement une forte biodiversité et une densité moyenne et 2) une association de foraminifères avec des espèces péritidales (36 à $48 \%$ ) représentées par Ammonia tepida, des Bolivinidae et des Rotaliina qui dominent sur les Miliolidae partout sauf dans la station 37 où les Miliolidae sont dominants.

Le contrôle saisonnier s'exprime surtout dans les bordures de la lagune, qui enregistrent durant l'été une augmentation de Loxoconcha au dépens de Cyprideis. De plus, la biodiversité et la densité de la bordure continentale sont affectées par les décharges fluviatiles de l'hiver. Par ailleurs, l'impact du stress anthropique s'exprime dans les stations proches des cages d'aquaculture et de la bordure continentale, en particulier durant les périodes d'été. Les teneurs élevées en éléments nutritifs engendrées par l'aquaculture, induisent une diminution des pourcentages de Loxoconcha (10, 5\%) et de Cyprideis (62\%) dans la station 31. L’impact des eaux usées de Oued Bouaroug (station 11 ) et de la station de lagunage (station 14) se traduit par une diminution du pourcentage de Loxoconcha au profit de celui de Cyprideis, la disparition des foraminifères épifauniques marins et l'augmentation des Spiroloculinidae. Enfin, dans la station 4, l'impact de la pollution qui est accentué durant l'été, se traduit par une diminution du pourcentage de Loxoconcha au profit de celui de Cyprideis.

La reconstitution des milieux écologiques pour la période 1000 à 1200 ans BP (Fig. 2 B) a montré que la station 14 se caractérisait par le milieu écologique III et que l'influence marine était plus importante dans les stations 37, 47 et 4 et plus faible dans les stations 31, 27 et 23. Ces résultats s'expliquent par le fait qu'il y a 1000 à 1200 ans, la limite interne de la lagune devait coïncider au moins avec la limite actuelle du marais salant de Taouima et la passe était située au niveau du double tombolo.

\section{Conclusions}

En plus du calcul des fréquences des associations de foraminifères benthiques et d'ostracodes et la reconstitution des milieux écologiques pour l'actuel (hiver 2003, été 2004) et pour une période ancienne (1000 à 1200 ans BP), ce travail a permis de : préciser le contrôle du stress anthropique et des facteurs naturels (climat, morphologie et taille de lagune, position de la passe, nature du substratum) et de confirmer la morphologie de la lagune et la position de la passe il y a 1000 ans BP. 
La connaissance de la Mer :

un vecteur du développement durable en Méditerranée
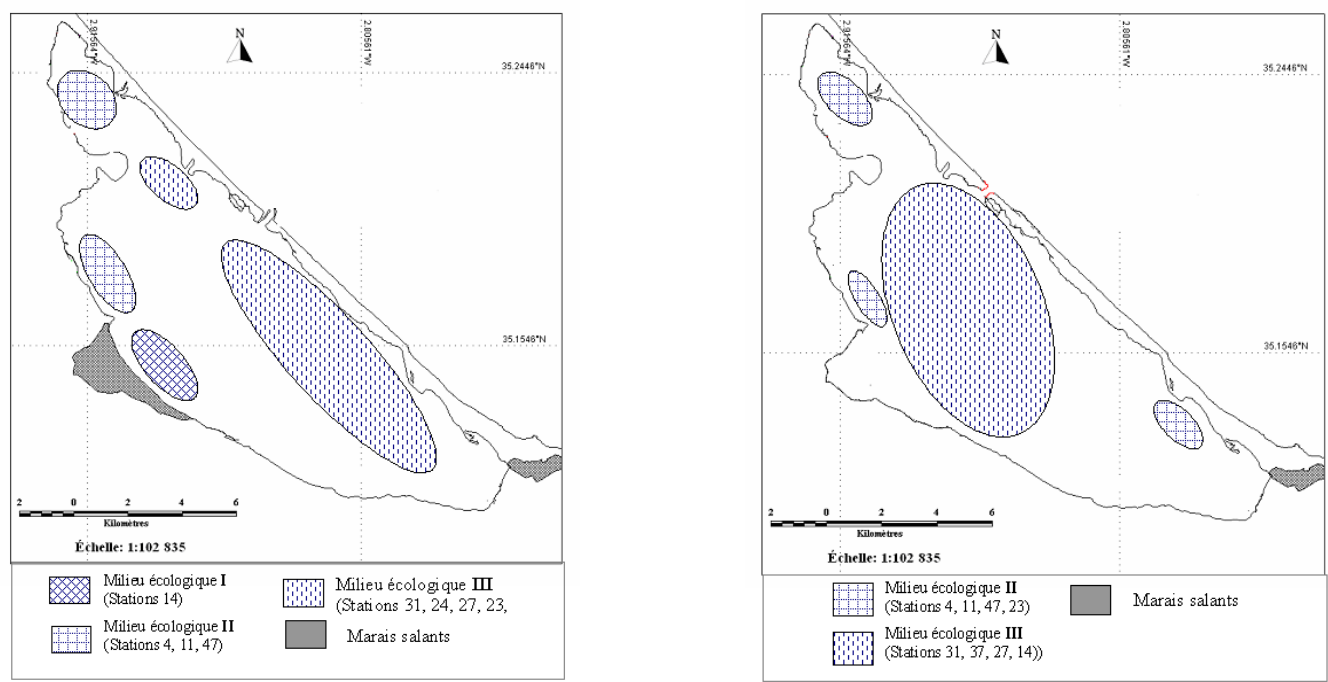

Fig.2: Les milieux écologiques de la lagune de Nador.

A gauche : Hiver 2003. A droite : 1000 à 1200 ans BP.

\section{Références bibliographiques}

BOUIMETARHAN I. (2004). L'écologie des foraminifères benthiques et des ostracodes dans la lagune de Nador. Mémoire de DESS. Univ. Med V-Agdal., Rabat.

GONZALEZ-REGALADO M.L., RUIZ F., ABAD M., TOSQUILLA J., HAMOUMI N., LABRAIMI M., BOUMAGGARD E.H., BOUIMETARHAN I., HSAIDA T., GUEDDARI F., TOUMI A., DAIS K.Y., BEN AHMED R. (2005). Distribución de los foraminíferos bentónicos en climas semiáridos: las lagunas de Nador (Marruecos) y El Meleh (Túnez). Geogaceta. 37, pp 211-214.

HAMOUMI N., HSAIDA T., TERHZAZ L., HAZIM M.E.A. (2005). Ecologie des Foraminifères benthiques et des Ostracodes de la lagune de Nador. Rapport scientifique, Partenaire P3 (Maroc), Projet COLASU.

IRZI Z. (1987). Etude sédimentologiques et micropaléontologique de la lagune de Nador (Maroc Oriental). Thèse $3^{\text {ème }}$ cycle, Univ. Pierre et Marie Curie, Paris VI, 172 p. IRZI Z. (2002). Les environnements du littoral méditerranéen oriental du Maroc compris entre l'oued Kiss et le Cap des Trois Fourches; Dynamique sédimentaire et étude d'impact des sites aménagés et l'analyse des associations de foraminifères benthiques de la lagune de Nador en relation avec leur écologie. Thèse de doctorat ès Sciences, Univ. Oujda, 290 p.

MAHJOUBI R. (2001). Nature et origine des flux de matière particulaire et son enregistrement dans un milieu paralique microtidal : cas de la lagune de Nador (Maroc nord oriental). Thèse Doctorat d'Etat ès Sciences, Univ Moulay Ismail, Meknès, 273 p. RUIZ F., ABAD M., GARCIA E.X.M., HAMOUMI N., LABRAIMI. M, BOUMAGGARD E.H., BOUIMETARHAN I. (2004). Los ostrácodos como indicadores ambientales: la laguna de Nador (NE de Marruecos). Geotemas, 6(2), pp 307-310. 